\title{
CRÍO-MICROSCOPÍA ELECTRÓNICA. RESOLVIENDO LA ESTRUCTURA MOLECULAR DE LA VIDA AL DETALLE ATÓMICO
}

Cryo-electron microscopy. Solving the molecular structure of life at the atomic detail

\author{
Alfredo Florez Ariza $^{1}$ y Daniel Guerra Giraldez ${ }^{2}$
}

\begin{abstract}
Resumen
Recientemente, la Crío-Microscopía Electrónica (CryoEM) ha alcanzado la resolución atómica para complejos moleculares de proteínas en solución acuosa. Tres contribuciones claves en este desarrollo fueron: establecer condiciones de energía mínima para no destruir las biomoléculas; la vitrificación de muestras a temperaturas ultrabajas para que las biomoléculas estén protegidas de la evaporación; el desarrollo de algoritmos para construir modelos tridimensionales a partir de imágenes CryoEM. Además, se propone la creación en la UPCH del primer centro de biología estructural en Perú, gracias a las colaboraciones ya establecidas con dos centros mundiales de referencia en CryoEM.
\end{abstract}

Palabras claves: Microscopía crio-electrónica, formación de imágenes, análisis de imagen, estructura molecular.

\begin{abstract}
Recently, the Cryo-Electron Microscopy technology (CryoEM) has reached atomic resolution for molecular complexes of proteins in aqueous solution. Three key contributions in this development were: setting conditions of minimal energy not to destroy the biomolecules; the vitrification of samples at ultra-low temperatures so that the biomolecules are protected from evaporation; the development of algorithms to build three-dimensional models from CryoEM images. Besides, it is proposed the creation in the UPCH of the first structural biology center in Peru, thanks to the collaborations already established with two world reference centers of CryoEM expertise.
\end{abstract}

Keywords: Cryo-electron microscopy, image formation, image analysis, molecular structure.

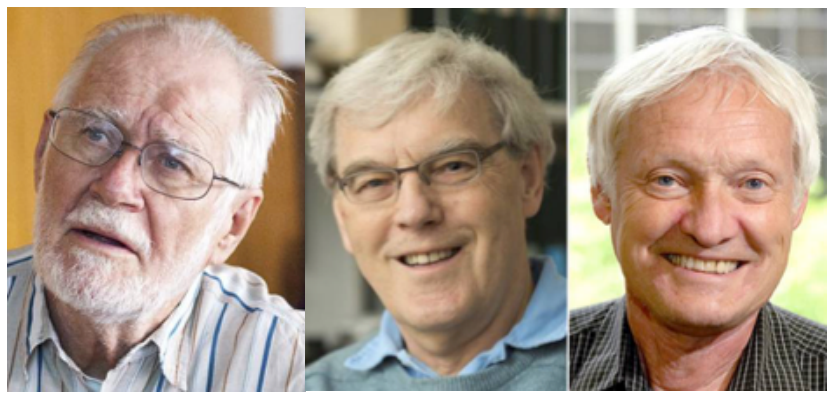

Jacques Dubochet, Richard Henderson y Joachim Frank. (FOTOS DE HTTPS: / / ELPAIS.COM / ELPAIS / 2017/ 10/04/ CIENCIA / 1507101567_361365.HTML)

a relación entre la estructura y la función
es necesaria y evidente en todos los
niveles de las ciencias de la vida. Así alas y manos como variaciones divergentes de un mismo patrón, las bio-máquinas que hacen funcionar a la célula están constituidas cada una por moléculas con la estructura adecuada para su propósito.

Las enzimas presentan la forma que recibe al sustrato y lo acomoda orientado a grupos

LNNano, CNPEM, Instituto de Física de São Carlos de la Universidad de São Paulo, Brasil.

Laboratorio de Moléculas Individuales, Facultad de Ciencias y Filosofía ACT, UPCH, Lima. 
electropositivos y electronegativos que aseguran que se lleve a cabo una reacción química. Las proteínas del citoesqueleto se forman como grandes vigas que pueden ensamblar redes con la rigidez o flexibilidad necesarias para soportar la estructura y movimiento de la célula. Los motores moleculares acoplan reacciones químicas y el uso de energía térmica para trasladarse en una dirección específica para, por ejemplo, transportar vesículas de material entre organelos.

La biología estructural plantea elucidar estos y muchos otros procesos observando la posición de cada átomo que forma la estructura funcional de estas macromoléculas de la vida. Este es el nivel en el que actúa la selección natural, al definirse si tras una mutación puntual una enzima conserva su actividad, es modificada, o si cambia la afinidad entre un ligando y un receptor. También a este nivel actúan todas las drogas y moléculas reguladoras, estableciendo uniones según la estructura dada por los átomos de la superficie de un receptor o blanco terapéutico en general. Por lo tanto, puede decirse que la biología estructural es un nivel de comprensión fundamental de la vida y resulta esencial para el desarrollo de tecnologías, como por ejemplo las farmacéuticas.

Desde la década de 1950, la difracción de rayos $X$ ha sido la técnica predominante para llevar a cabo esta tarea, pues proveyendo un cristal formado por repeticiones periódicas y bien organizadas de la molécula en estudio, es posible elucidar su estructura 3D con resolución atómica. Sin embargo, para muchas de estas moléculas existe una gran dificultad de formar cristales, aun utilizando muestras altamente concentradas $(20 \mathrm{mg} / \mathrm{mL})$. Por otro lado, la resonancia magnética nuclear (NMR, por sus siglas en inglés), se basa en la detección de los átomos individuales de la molécula mediante la excitación de sus núcleos por un fuerte campo magnético con el que interactúan según su desplazamiento químico; sin embargo, cuando las moléculas (por ejemplo, proteínas) son más grandes que 35-50 kDa, las señales de desplazamiento químico de los núcleos se superponen entre sí, tornando imposible el problema de diferenciarlos. Estas limitaciones motivaron la búsqueda de técnicas alternativas para esclarecer la estructura de las macromoléculas biológicas. El premio Nobel 2017 de Química fue otorgado a Richard Henderson, Jacques Dubochet y Joachim Frank por haber logrado aportes definitivos en el uso de la microscopía electrónica en biología estructural. En una historia llena de ingenio y visionario espíritu científico se fueron superando barreras conceptuales y técnicas hasta llegar hoy día a ofrecer la misma calidad que la cristalografía de rayos $X$, con la ventaja de no requerir la cristalización de la muestra y con la capacidad, en algunos casos, de dar información directa sobre diferentes estados conformacionales asociados a la función biológica de un complejo molecular.

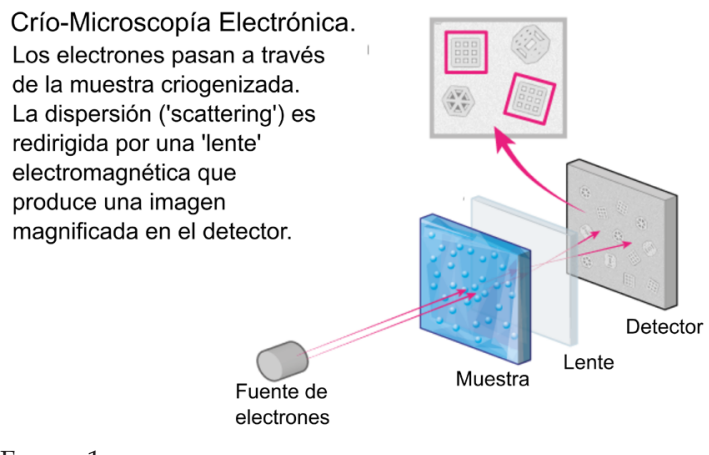

FIGURA 1

¿QUÉ ES LA CRÍO-MICROSCOPÍA DE TRANSMISIÓN ELECTRÓNICA?

El microscopio de transmisión electrónica (TEM, por sus siglas en inglés) produce una señal a partir de un haz de electrones que es dispersado por su interacción con la muestra 
en estudio y recogido por un detector que produce imágenes bidimensionales. El voltaje de aceleración típico $(100-300 \mathrm{kV})$ confiere a los electrones una velocidad relativística (i.e. significativamente cercana a la velocidad de la luz) para la que se calcula una longitud de onda

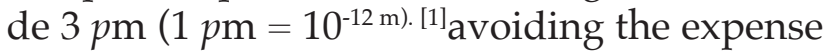
of high-voltage microscopy but providing the possibility of atomic resolution even in the absence of lens-aberration correction. For specimens thicker than a few tens of nm, the image intensity and scattering contrast are likely to be higher than at lower voltage, as is the visibility of ionization edges below $1000 \mathrm{eV}$ (as required for EELS elemental analysis Esta pequeñísima longitud de onda permite un límite teórico de resolución diminuto, mucho más allá de los $200 \mathrm{~nm}$ de la microscopía de luz visible o de los $20 \mathrm{~nm}$ alcanzados con las últimas técnicas de localización por fluorescencia. ${ }^{[2]}$ Dado que los átomos tienen tamaños varias veces más grandes que este límite (el átomo de hidrógeno mide aproximadamente 10-10 $\mathrm{m})$, ya por principio TEM debería resultar una técnica óptima para observar estructuras moleculares con resolución atómica. Imagen tomada de la ref. bibliog ${ }^{[3]}$.

Richard Henderson fue pionero en buscar la aplicabilidad de TEM para elucidar la estructura de moléculas biológicas. Determinó que uno de los problemas principales que debían superarse era la cantidad de energía transmitida por el haz de electrones, que es tan alta que puede destruir la muestra en estudio. Utilizando pulsos de baja energía, sus exploraciones rindieron fruto en 1975 con su trabajo sobre la bacteriorodopsina. Esta proteína está naturalmente embebida en la membrana celular de halobacterias, en donde genera energía a partir de la captación de luz. Resultaba imposible conservar bacteriorodopsina en estado nativo, pura y concentrada para su cristalización y análisis por difracción de rayos X. Por esto, utilizaron un cristal bidimensional, que contenía a la proteína en su estado nativo, y lo colocaron en el microscopio electrónico de transmisión. La información generada por el patrón de difracción del haz de electrones, luego de posicionar el cristal en diferentes orientaciones angulares, permitió obtener un modelo 3D de la bacteriorodopsina. La resolución alcanzada fue de $7 \AA$, suficiente para mostrar cómo las siete hélices que conforman la proteína se extienden de manera perpendicular al plano de la membrana en la que está inmersa.[4]

Este temprano antecedente, aunque alentador, no resolvía la situación para la mayoría de moléculas biológicas. Dado que el haz de electrones es sumamente sensible, todo el proceso de microscopía de transmisión debe llevarse a cabo en alto vacío. Como el agua se evapora en estas condiciones y la estructura nativa de las biomoléculas depende justamente de interacciones con el solvente acuoso, el valor informativo de la muestra se perdería totalmente en una típica toma de imágenes por TEM.

Se propuso entonces que a temperaturas muy bajas se podría proteger la estructura nativa, que quedaría atrapada en agua vitrificada. Ante un congelamiento muy rápido, las moléculas de agua de la solución en la que se encuentra la macromolécula en estudio no tienen el tiempo suficiente para reorganizarse y formar estructuras cristalinas regulares -hielo hexagonal o cúbico-, y por el contrario, pasan a formar un estado de agua vítrica o hielo amorfo. $[2,3]$ Este estado sólido-amorfo del agua, semejante al que presenta en estado líquido, impediría la rápida evaporación, conservando a la biomolécula en su estado nativo y a la vez proporciona una parcial protección frente a la radiación electrónica. Ya en 1960, el científico venezolanoHumberto Fernandez-Morán había 
demostrado la aplicación del helio líquido en la preparación de muestras biológicas para la microscopía electrónica. ${ }^{[5]}$ Años más tarde, Jacques Dubochet y su equipo logró conservar virus mediante la inmersión en etano líquido a $-190^{\circ} \mathrm{C}$, y fue posible visualizar por primera vez cómo las partículas individuales quedaban capturadas en su estado nativo dentro de agua vitrificada, en múltiples y diferentes orientaciones espaciales. ${ }^{[6]}$

Procedimiento DE PREPARACIÓN DE MUESTRA PARA CRYoEM. Tomado de la ref. bibliog. ${ }^{[7]}$

Las imágenes bidimensionales así obtenidas de una biopartícula en múltiples orientaciones ya contenían en conjunto toda la información necesaria para construir un detallado modelo de la estructura 3D. Sin embargo, este complejo proceso matemático necesitaba aun del aumento de la capacidad de cómputo y

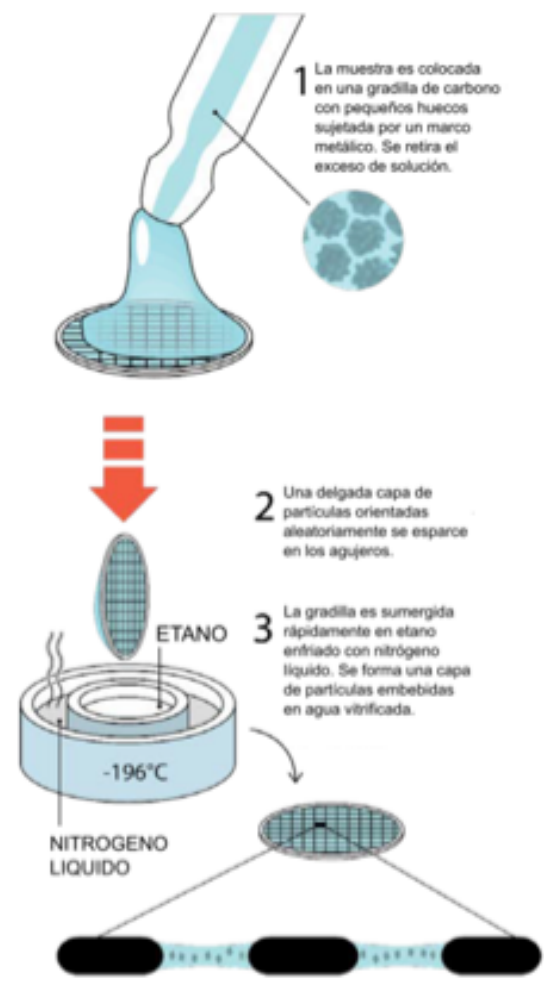

FIGURA 2 el desarrollo de ingeniosos algoritmos en los que la contribución de Joachim Frank ha sido destacada.

ANÁlisis DE IMÁGENES PARA LA OBTENCIÓN DE ESTRUCTURAS 3D. Tomado de la ref. bibliog. ${ }^{[7]}$

Actualmente, este proceso se inicia con la identificación de las biopartículas como imágenes individuales de la proteína o complejo molecular que será analizado a partir de la imagen de todo el campo de visión del microscopio. Estas partículas, en un número típico de decenas o cientos de miles, son extraídas y clasificadas según las similitudes que muestran entre sí. Mediante algoritmos de correlación, se agrupa las imágenes en un grupo de clases (algunos miles), cada

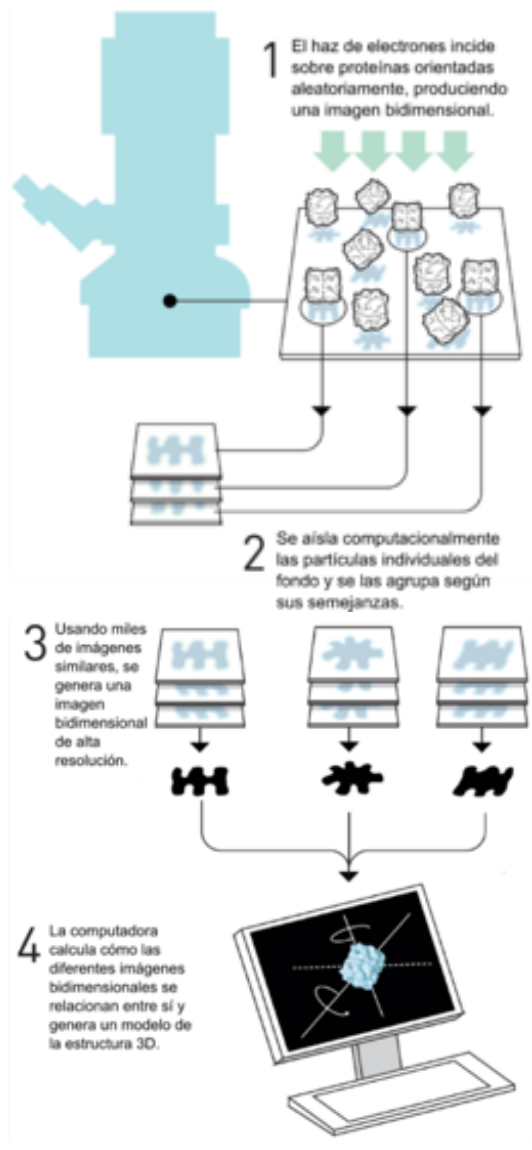

FIGURA 3 
una conformada por numerosas partículas individuales. La información de las imágenes individuales es sumada dentro de su clase, para finalmente dar lugar a lo que se conoce como "clase promedio" o "classum". Un classum es la imagen bidimensional que muestra una orientación espacial específica de la partícula en estudio, y que, debido a que está generada por la suma de información de varias partículas, tiene una mayor relación señal/ruido que sus componentes individuales. Asumiendo que cada classum representa una proyección de la partícula tridimensional, se emplean diversos algoritmos para calcular las orientaciones angulares o "ángulos de Euler" que estas proyecciones tendrían en el espacio, y así construir un modelo 3D del objeto completo. A partir de este punto se inician iteraciones de refinamiento, en las que el primer modelo 3D se usa para generar computacionalmente nuevas proyecciones bidimensionales, las cuales son útiles para volver a analizar las micrografías originales, lo que permite obtener nuevos y mejores classum. Con varios ciclos de análisis se mejora progresivamente el modelo 3D. ${ }^{[8]}$ En ocasiones, el proceso permite identificar diferentes estados conformacionales y elucidar así la dinámica de acción de un complejo molecular, incluso a partir de un solo set de micrografías. ${ }^{[9]}$

Desde su origen hasta la actualidad: El enorme potencial de la Crío-Microscopía Electrónica.

Los primeros logros en CryoEM recibieron el apodo de ciencia de "blobology", por producir imágenes de baja resolución (15-20 Å) con la forma de globos con protuberancias suaves, a diferencia de la alta resolución conseguida por cristalografía de rayos $X(1,7-3,1 \AA)$ que permite identificar átomos individuales. Sin embargo, las continuas mejoras instrumentales e informáticas condujeron a una situación clave el año 2013. Es entonces que se inicia la llamada "revolución de la resolución", impulsada por a la nueva generación de detectores directos de electrones que presentan una velocidad y sensibilidad muy superior a los dispositivos CCD utilizados anteriormente que requerían convertir previamente los electrones en fotones. Con una mejor relación señal/ruido y la capacidad de producir películas en lugar de tomas aisladas, en los últimos años la tecnología CryoEM ha alcanzado la resolución de la tradicional cristalografía de rayos $X[1,6]$ $y$ ha superado su eficiencia, ya que no requiere cristalizar las muestras. ${ }^{[10]}$

Algunos trabajos destacados utilizando CryoEM Y EL ANÁLISIS DE PARTÍCULAS INDIVIDUALES

En la Figura 4.

(A) En el 2015 se logró resolver la estructura del virus del Zika a 3,8 Å de resolución ${ }^{[11]}$; el triángulo señala la unidad asimétrica.

(B) Menos de un año después, Merk y colaboradores consiguieron superar la barrera hasta entonces infranqueable de los $2 \AA$ Å de resolución, al elucidar la estructura 3D de la enzima glutamato deshidrogenasa a 1,8 $\AA$ de resolución, demostrando la

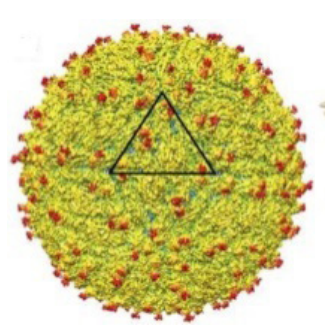

A

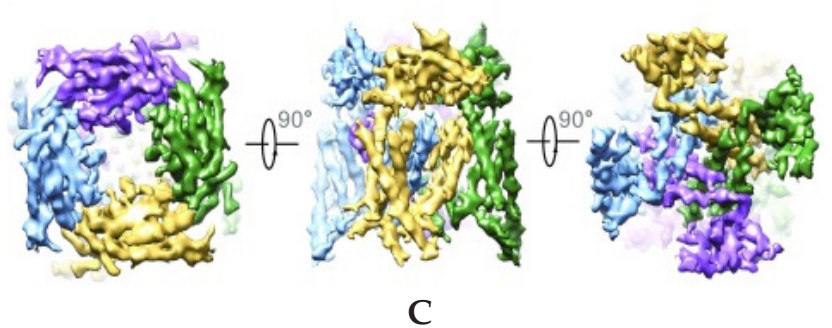

FIGURA 4.

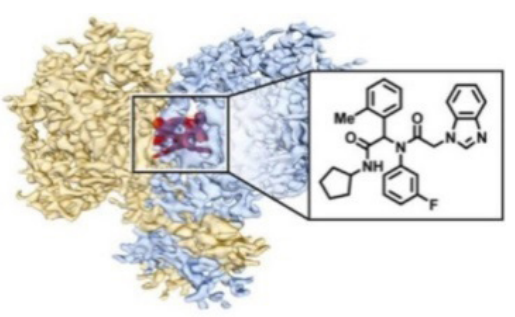

B
C 
posibilidad del diseño de fármacos a partir de estructuras reveladas por CryoEM ${ }^{[12]}$; se señala el inhibidor ML309.

(C) En octubre 2017, la estructura a 3,6 de resolución de una proteína de $66 \mathrm{kDa}$ (TRPML1, un receptor de canal endolisozimal) demostró que aun proteínas pequeñas pueden ser estudiadas con esta tecnología. ${ }^{[13]}$

CRYoEM en la UPCH: Propuesta del pRIMer ‘hub’ de biología estructural en el Perú

Los costos de equipamiento, operación y mantenimiento necesarios para el uso de CryoEM son muy altos, de manera que solo una institución que coopera con muchos usuarios es capaz de aprovechar la inversión mediante una plataforma de uso común para diversas líneas de investigación. Esta es la estrategia del Laboratorio Nacional de Nanotecnología, LNNano, del Centro Nacional de Pesquisa em Energia e Materiais de Brasil que ha formado un equipo de alto nivel bajo la dirección de Rodrigo Portugal y la asesoría de Marin van Heel, quien ha recibido los premios Ernst Ruska 1985 y Wiley 2017 por sus contribuciones determinantes en la algoritmia de análisis. [14] El laboratorio de Moléculas Individuales de la Facultad de Ciencias de la UPCH coopera cercanamente con LNNano para la caracterización del complejo de transcripción de la bacteria Escherichia coli y su mecanismo de regulación en situaciones de estrés relacionadas a la virulencia y resistencia al ataque inmune y antibiótico. De la misma manera, nuestro equipo investiga la dinámica de transcripción de Mycobacterium tuberculosis y su interacción con nuevos antibióticos mientras realizamos los estudios estructurales mediante CryoEM en colaboración con Carlos Bustamante y Eva Nogales de la Universidad de California en Berkeley. Estas investigaciones no solo están realizando avances importantes en su campo, sino que además prometen abrir el puente para el desarrollo de la biología estructural en el Perú mediante la inversión inteligente en cooperación.

Cualquier investigación bioquímica que conduzca al aislamiento de complejos moleculares puros, podría producir muestras en condiciones adecuadas para la toma de imágenes por TEM en pocos días. Pasada esta etapa, que puede implicar un viaje muy breve de pocos días, o el simple envío de muestras estabilizadas en gradillas de carbono, un equipo de investigadores peruanos podría superar el siguiente paso limitante: el análisis de imágenes. Un clúster de computadoras relativamente modesto, con una capacidad de almacenamiento de datos mediana, involucra una inversión del orden de 10-20 mil dólares (en lugar de los varios millones de costo del equipo TEM adecuado) y podría suplir a diversos investigadores del país, creando una dinámica de alto rendimiento al servicio de la ciencia peruana.

\section{Propuesta de un Centro de biología ESTRUCTURAL EN LA UPCH}

Las investigaciones en biodiversidad, microbiología, medicina u otras áreas identifican complejos moleculares de interés. Las muestras son preparadas con pureza y calidad mínimas para estudios en CryoEM a realizarse en colaboración en Campinas y Berkeley. Las imágenes producidas son enviadas a la UPCH para su análisis y la construcción de modelos estructurales. La biología estructural permite el desarrollo de ingeniería de proteínas, diseño y mejora de ligandos y potencia la investigación en general para el servicio de la sociedad.

Además deconsiderarsuimportancia científica, debemos reconocer que en el Perú los esfuerzos 


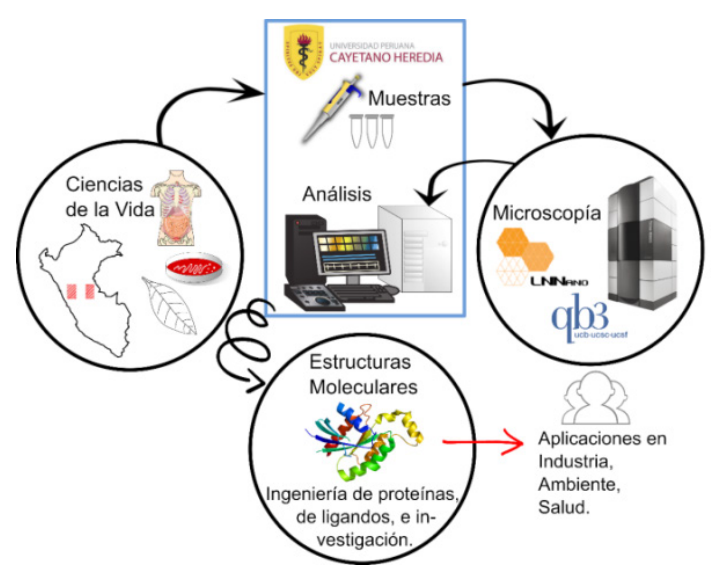

FIGURA 5

en biotecnología se encuentran severamente limitados por la incapacidad de realizar la caracterización estructural de complejos moleculares de interés. Actualmente, las ciencias genómicas han potenciado el hallazgo de nuevas moléculas de importancia industrial, ambiental y biomédica, sin embargo, tras su descubrimiento es necesario pasar por etapas de caracterización bioquímica y estructural que puedan conducir a su aplicabilidad. La ingeniería de proteínas y el diseño racional de ligandos o drogas son disciplinas que se basan en la biología estructural para desarrollar productos patentables de alto impacto y valor. Gracias a las colaboraciones establecidas en los últimos años, se nos presenta hoy la oportunidad de protagonizar el nacimiento de la biología estructural en el Perú. El tiempo apremia.

\section{REFERENCIAS BibLIOGRÁficAS}

1. Egerton RF. Choice of operating voltage for a transmission electron microscope. Ultramicroscopy. 2014; 145:85-93.

2. Guerra DG. Superando el límite infranqueable de la microscopía a la nanoscopía. Acta Hered. 2015; 55:72.

3. Cressey D and Callaway E. Cryo-electron microscopy wins chemistry Nobel. Nat News. 2017; 550(7675):167.

4. Henderson R and Unwin PN. Three-dimensional model of purple membrane obtained by electron microscopy. Nature. 1975;257(5521):28.

5. Fernandez-Moran H. Low-temperature preparation techniques for electron microscopy of biological specimens based on rapid freezing with liquid helium II. Ann N Y Acad Sci. 1960; 85:689-713.

6. Dubochet J, Adrian M, Lepault J and McDowall AW. Emerging techniques: Cryo-electron microscopy of vitrified biological specimens. Trends Biochem Sci. 1985;10(4):143-146.

7. The Nobel Prize in Physics 2017 - Scientific Background: The development of cryo-electron microscopy". Nobelprize.org. Nobel Media AB 2014. Web. 16 Jan 2018. <http: / / www.nobelprize. org / nobel_prizes / chemistry / laureates / 2017 / advanced.html>

8. van Heel MG. Image formation and image analysis in electron microscopy. Groningen: s.n., $1981.147 \mathrm{p}$.

9. Roh $\mathrm{SH}$ et al. Subunit conformational variation within individual GroEL oligomers resolved by Cryo-EM. Proc Natl Acad Sci USA. 2017;114(31):8259-8264.

10. van Heel $M$ and Schatz $M$. Reassessing the revolutions resolutions. bioRxiv. Nov 2017. doi: https:/ / doi.org/10.1101/224402

11. Sirohi $\mathrm{D}$ et al. The $3.8 \AA$ resolution cryo-EM structure of Zika virus. Science. 2016; 352(6284):467-470.

12. Merk Aet al. Breaking cryo-EM resolution barriers to facilitate drug discovery. Cell. 2016;165(7):16981707.

13. Zhang S, Li N, Zeng W, Gao N and Yang M. CryoEM structures of the mammalian endo-lysosomal TRPML1 channel elucidate the combined regulation mechanism. Protein Cell. 2017;8(11):834-847.

14. The 16th Annual Wiley prize in biomedical sciences awarded for pioneering developments in electron microscopy. Wiley News Room - Press Releases, News, Events \& Media. URL disponible en: http://newsroom.wiley. $\mathrm{com} /$ press-release / all-corporate-news / 16thannual-wiley-prize-biomedical-sciencesawarded-pioneering-deve. (Fecha de acceso: 16ene-2018).

\section{CORRESPONDENCIA:}

daniel.guerra@upch.pe 